\title{
Multiply Modified Repeating DNA Templates for Production of Novel DNA-based Nanomaterial
}

Liat Katrivas, ${ }^{[a]}$ Benjamin Kempinski, ${ }^{[a]}$ Kfir Kuchuk, ${ }^{[b]}$ Uri Sivan ${ }^{[b]}$ and Alexander Kotlyar*[a]

a Department of Biochemistry and Molecular Biology, George S. Wise Faculty of Life Sciences and the Center of Nanoscience and Nanotechnology, Tel Aviv University, Ramat Aviv, Tel Aviv 69978, Israel

${ }^{b}$ Department of Physics and the Russell Berrie Nanotechnology Institute, Technion - Israel Institute of Technology, Haifa, 3200003, Israel

*Corresponding Author Email: s2shak@tauex.tau.ac.il 


\section{Content}

Sequences of the oligonucleotides (Table S1) ............................................................... 3

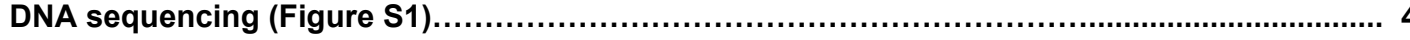

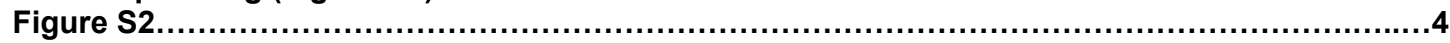

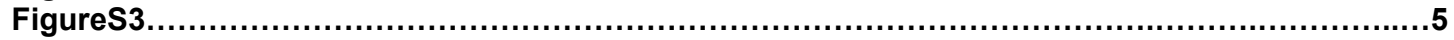

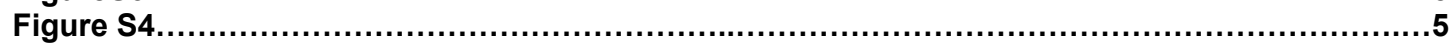

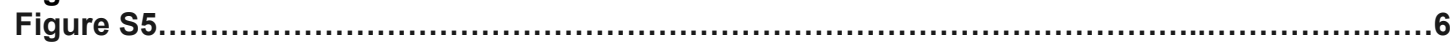

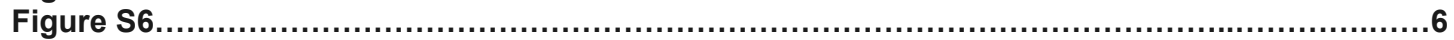

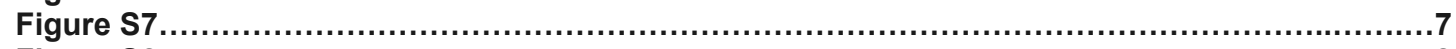

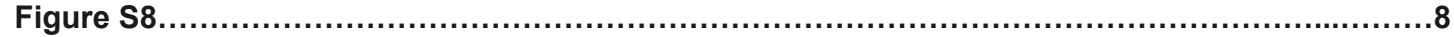

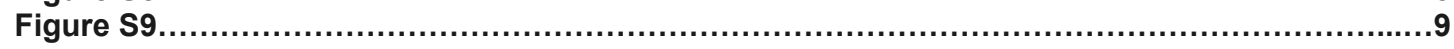

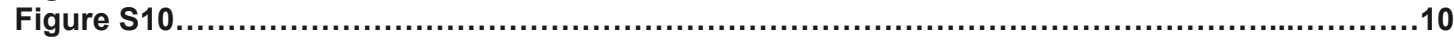

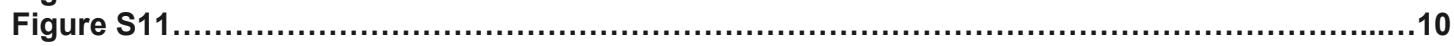

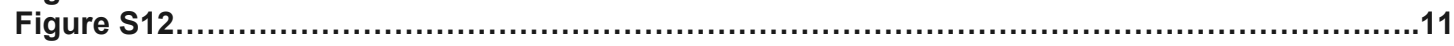

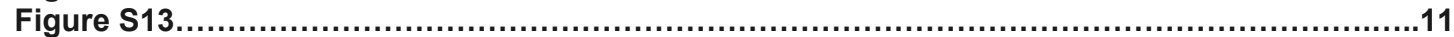

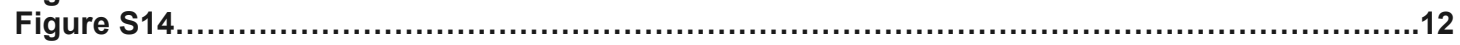


Table S1: Oligonucleotide sequences and their extinction coefficients.

\begin{tabular}{|c|c|}
\hline Number and sequence of oligonucleotide & $\begin{array}{l}\text { Extinction coefficient, } \\
\qquad \mathrm{mM}^{-1} \mathrm{~cm}^{-1}\end{array}$ \\
\hline 1. 5'-Pi-ACGATGACGA-3' & 137.9 \\
\hline 2. $\quad$ 5`-Pi-ATCGTTCGTC-3' & 119.6 \\
\hline 3. $5{ }^{\circ}-\mathrm{Pi}-\mathrm{ATCGTAAGATCGGCAGTTCCTTGCG-3`}$ & 316.9 \\
\hline 5`-Pi-ATCGTAAGATCGGCAGTTCCTTGCG-Biotin-3` & 316.9 \\
\hline 5. $5{ }^{\prime}-$ CGCAAGGAACTGCCGATCTT-3` $^{`}$ & 260.7 \\
\hline 6. $\quad 5 `$-Pi- GTTAGG-3` & 75.7 \\
\hline 7. $\quad 5-\mathrm{Pi}-\mathrm{AACCCT}-3^{\prime}$ & 78.8 \\
\hline $8 . \quad$ 5"-Pi- ACGATCAGACATCAG-3' & 203.9 \\
\hline 5"-Pi-ATCGTCTGATGTCTG-3' & 181.6 \\
\hline 10. $\quad 5^{\prime \prime}-\mathrm{Pi}-\mathrm{ATCGTCTGA}\left[{ }^{*} \mathrm{dT}-\mathrm{NH}_{3}\right]$ GTCTG-3' & 181.6 \\
\hline 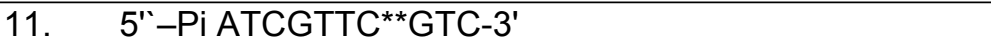 & 119.6 \\
\hline 5'-AC'mGATGACmGA-3' & 137.9 \\
\hline 13. 5 '-Pi -ACGATGATGA-3' & 134.7 \\
\hline 14. 5 '-Pi-ATTGTTCGTC-3' & 116.4 \\
\hline
\end{tabular}

* Amino group attached to $5^{\text {th }}$ position of the thymine ring via a 6 -carbon spacer. The oligonucleotide was from Bio-Synthesis Inc. (Lewisville, TX, USA).

** Phosphorothioate residue. The oligonucleotide was from Integrated DNA Technologies, Inc. (Coralville, IA, USA)

$\mathrm{C}^{\mathrm{m}}$ - 5-Methylcytosine

Deoxyguanosine $(G)$, deoxythymidine $(T)$, deoxycytidine $(C)$, deoxyadenosine $(A)$ 


\section{DNA sequencing}

The molecules were produced from half-complementary olygonucleotides, 5'- $\mathrm{Pi}$ ACGATGACGA-3' and 5'-Pi- ATCGTTCGTC -3', as described in Materials and Methods. DNA Sanger sequencing was performed with $A B I 3500 x I$ Genetic Analyzer Sequencer using BigDye Terminator Cycle Sequencing Kit from AB. Custom primers that match the initiator sequence were used in PCR amplification to generate fluorescent-labeled fragments. The labeled fragments were separated on the capillary sequencer; an internal size standard was used in each capillary. GeneScanT 600 LIZ® Size Standard is the custom size standard used and the data were analyzed by $A B$ GeneScan software.

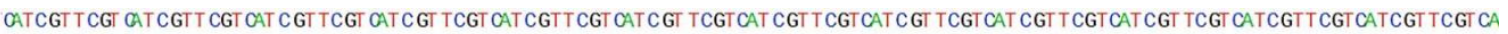

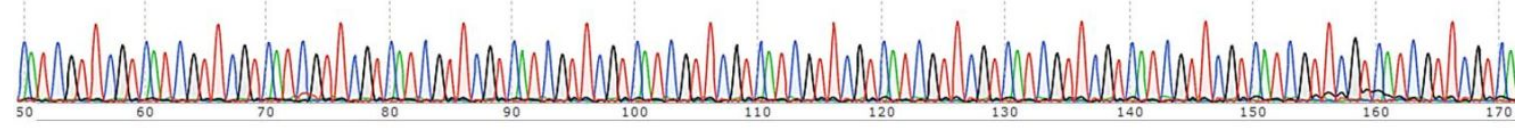

ГCATCGTTCGT CATCGT TCGT CATCGT TCGT CATCGTTCGT CATC GT TCGT CATCGTTCGTCATCGTTCGT CATC GT TCGT CATCGTTCGTCATCGT TCGT CATCGT TCGTCATCGTTCGT CATCGTTCGTCA

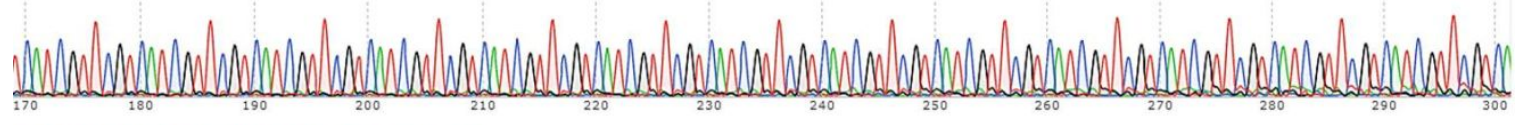

TCATCGITCGTCATCGI TCG CATCGTTCGTCATCGTTCGT CAT

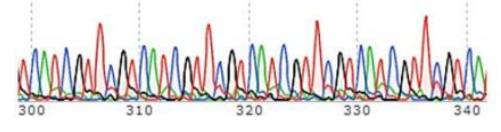

Figure S1. Sanger sequencing results of the DNA extension product.
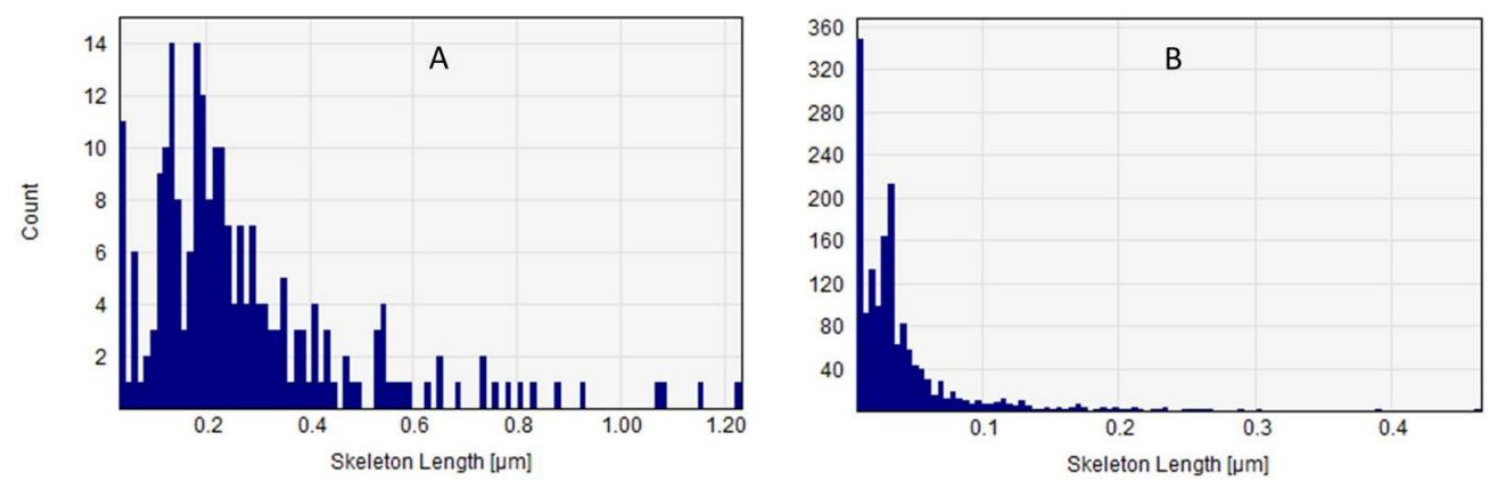

Figure S2. Statistical length imaging analysis of the molecules presented in Figures $3 \mathrm{~A}(\mathrm{~A})$ and 3B (B) using SPIP ${ }^{\text {TM }}$ software. 

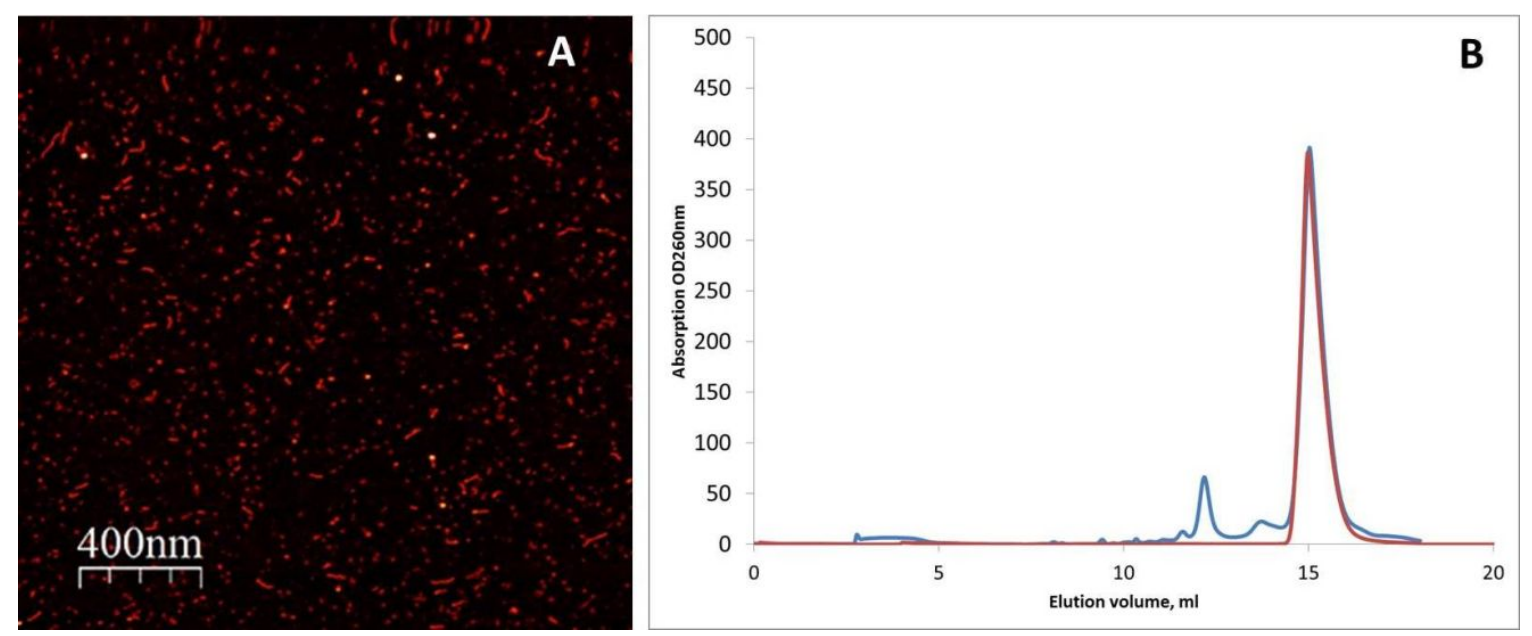

Figure S3. A - AFM image of DNA molecules, synthesized from a pair of half-complementary decameric unpurified oligonucleotides: 5'-Pi-ACGATGACGA-3' and 5'-Pi-ATCGTTCGTC3'. The DNA synthesis and AFM imaging were as described in Materials and Methods; B HPLC analysis of unpurified (blue curve) and HPLC-purified (red curve) 5'-PiACGATGACGA-3' oligonucleotide. The oligonucleotide was purified on an ion-exchange PolyWAX $1000 \AA$ column as described in Materials and Methods.
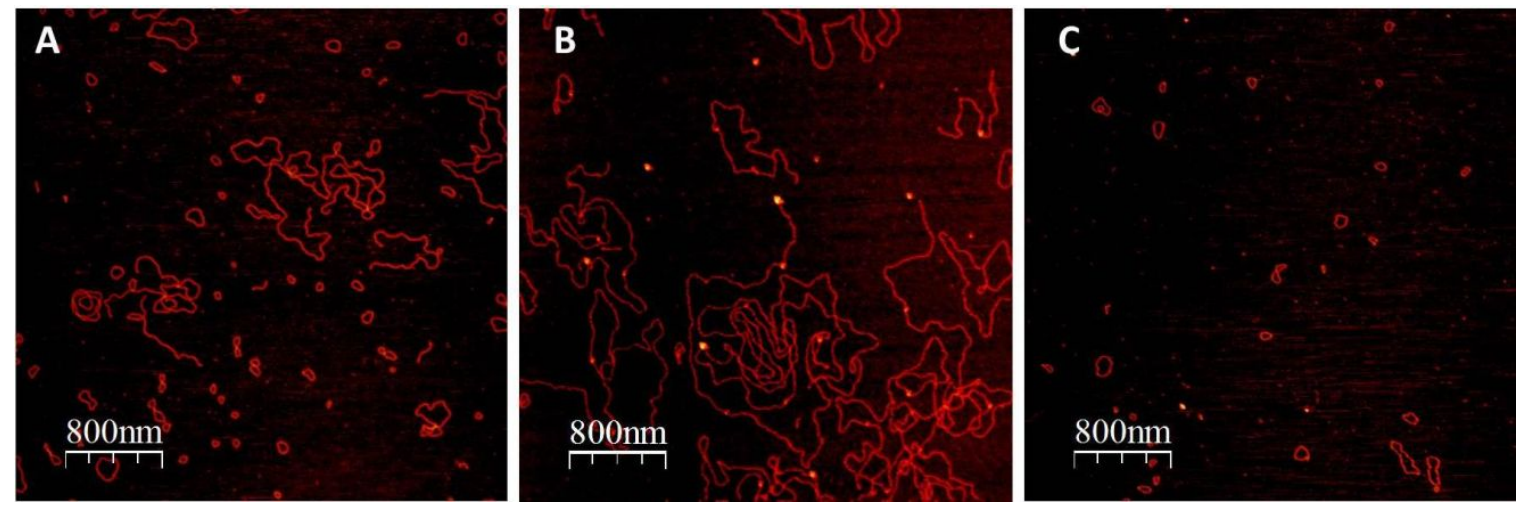

Figure S4. AFM images of DNA molecules assembled from a pair of purified halfcomplementary decameric oligonucleotides: 5'-Pi-ACGATGACGA-3' and 5'-PiATCGTTCGTC-3'. The DNA was synthesized from the HPLC-purified oligonucleotides as described in Materials and Methods. The synthesized molecules (A) were separated on a size-exclusion Sepharose CL-2B column at neutral $\mathrm{pH}$. The void volume fraction $(\mathrm{B})$ and the fraction eluted after the void volume $(\mathrm{C})$ were imaged by AFM as described in Materials and Methods. 


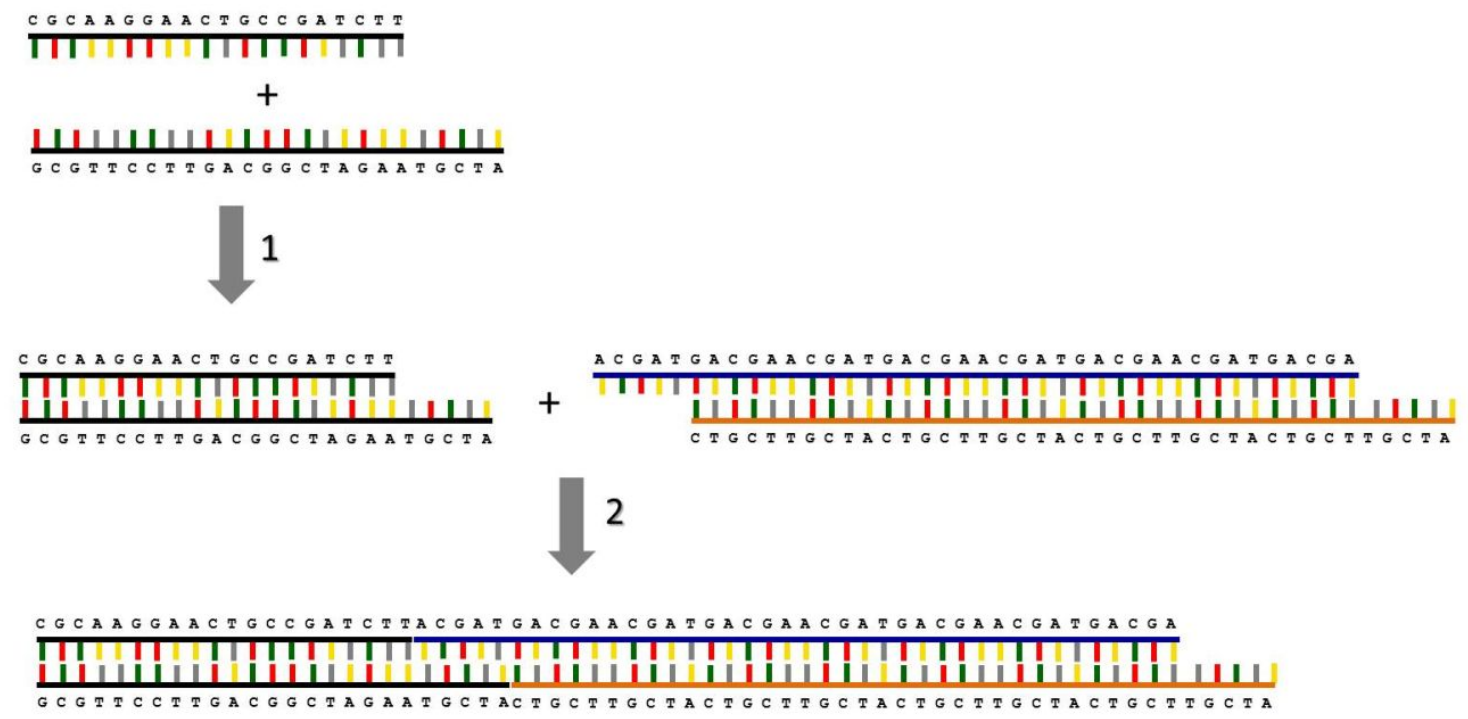

Figure S5. Schematic illustration of termination of DNA extension by a stopper. 1 Formation of a stopper from 25- and 20-base oligonucleotides. The stopper consists of a 20 bp fragment and a 5-base phosphorylated overhang at the 5'-end of the duplex. 2 Hybridization of the stopper's overhang with the DNA formed during the assembling of halfcomplementary oligonucleotides. The resulting DNA has a blunt and a sticky end and can be extended only from one (sticky) side.

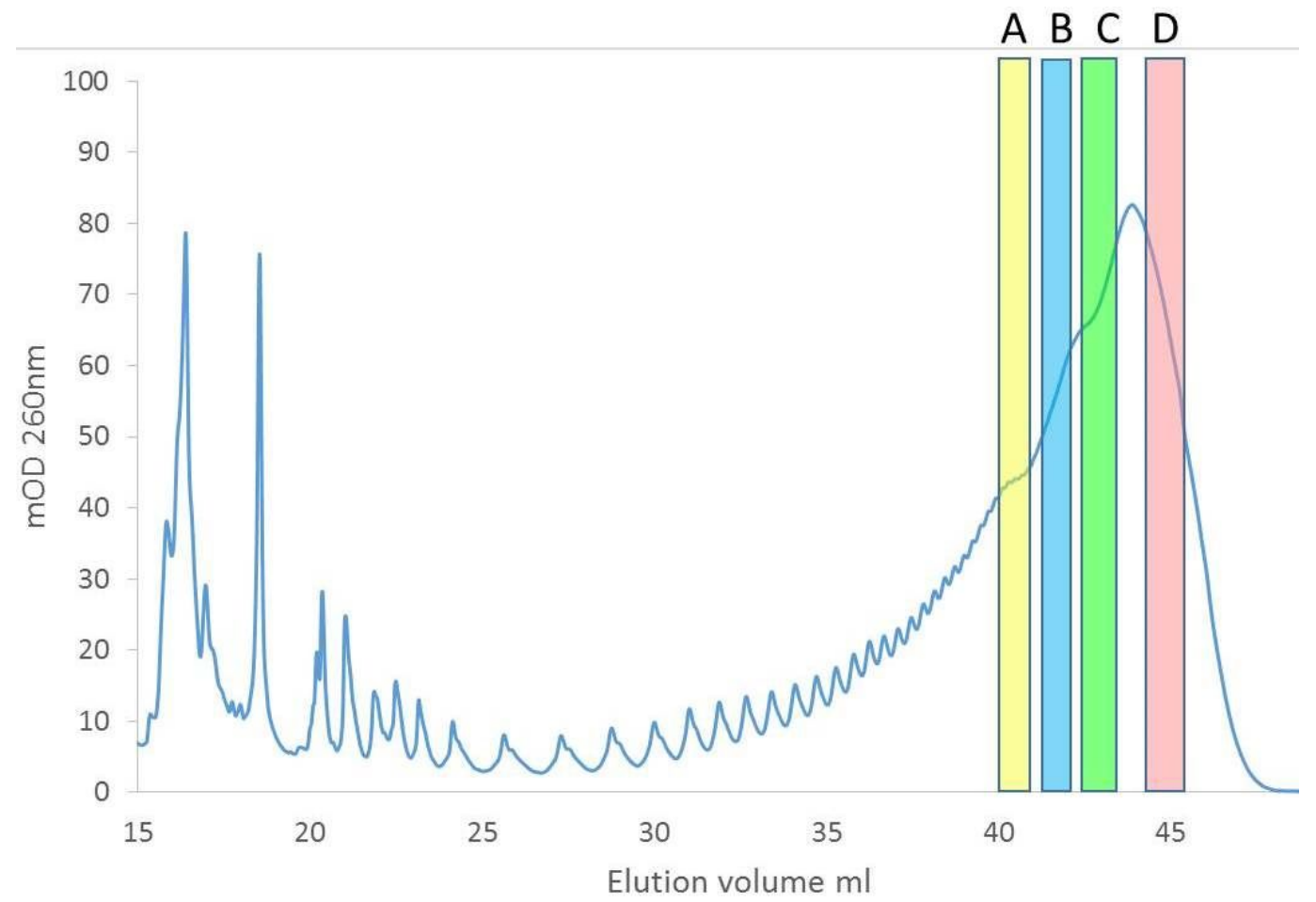

Figure S6. Separation of the synthesized dsDNA molecules by ion-exchange HPLC. The molecules were synthesized from a mixture of half-complementary decameric oligonucleotides, 5'-Pi-ACGATGACGA-3' and 5'-Pi-ATCGTTCGTC-3', together with stopper-forming oligonucleotides, 5-Pi-ATCGTAAGATCGGCAGTTCCTTGCG-3' and 5'CGCAAGGAACTGCCGATCTT-3', as described in Materials and Methods. The molar ratio 
between the former (half-complementary) and the latter (stopper-forming) oligonucleotides was 1 to 40. The synthesis products were separated on a TSKgel DNA-NPR column as described in Materials and Methods. The fractions (A, B, C, and D) were analysed by AFM and electrophoresis (see Figure 4).

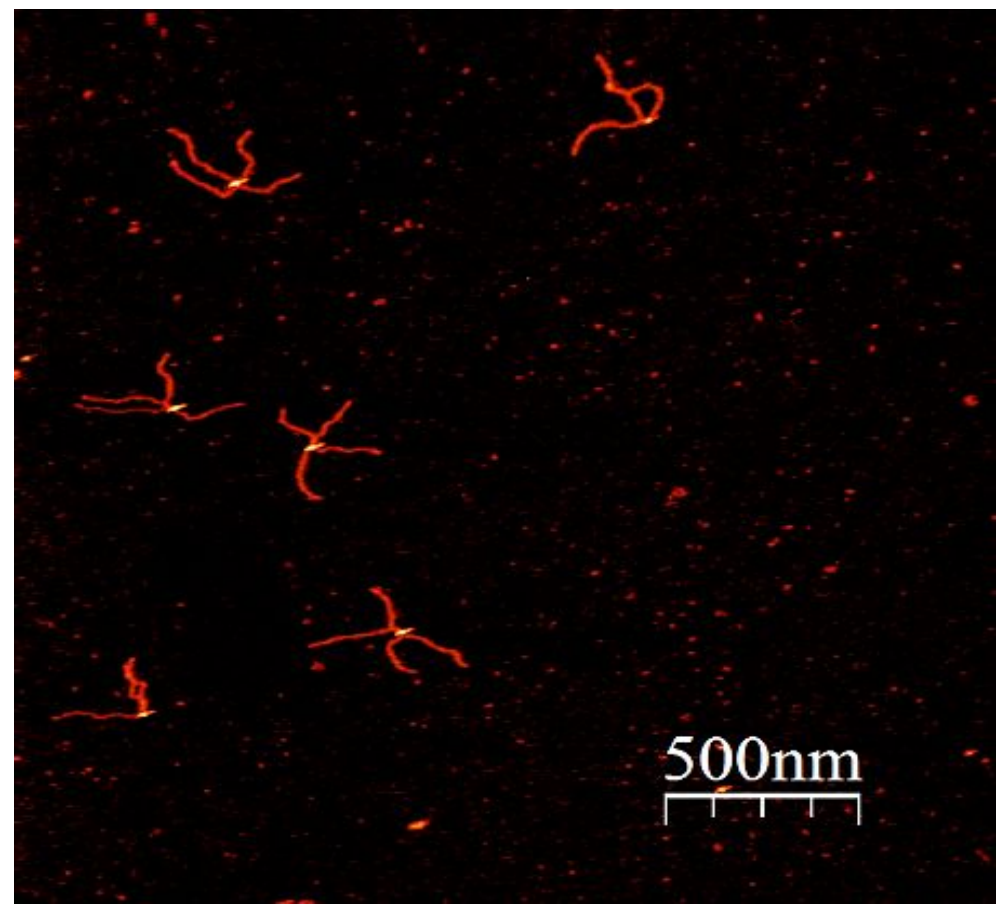

Figure S7. AFM image of conjugates between biotin-functionalized DNA and avidin. The DNA molecules ( 700 bp) were prepared from oligonucleotides: '1', '2', '4' and '5' (see Table S1) and purified by HPLC as described in Materials and Methods. The DNA-avidin conjugates were prepared and imaged by AFM as described in Materials and Methods. 


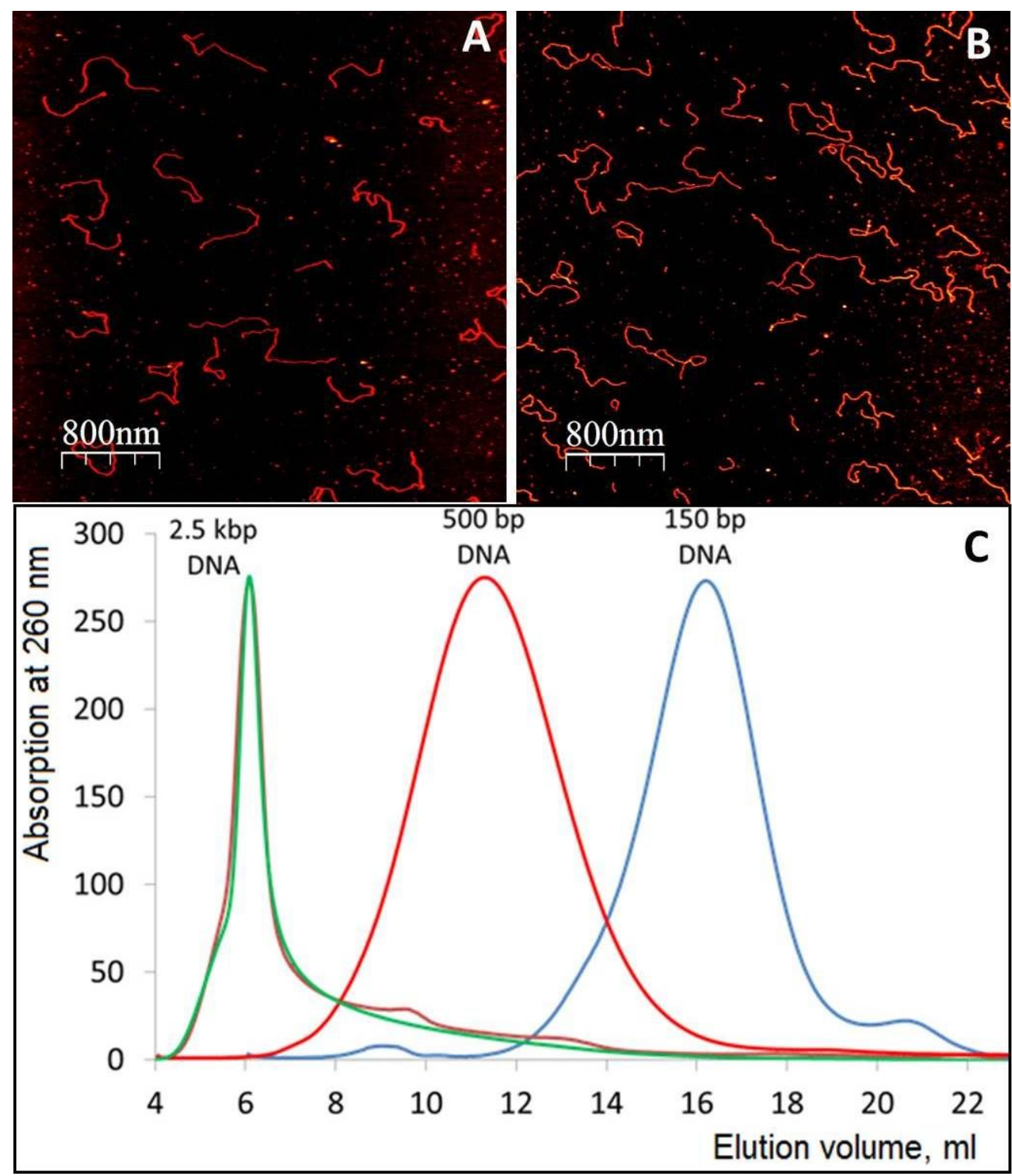

Figure S8. Size-exclusion chromatography analysis of strands composing dsDNA. A - AFM image of DNA molecules synthesized from a pair of half-complementary decameric oligonucleotides, 5'-Pi-ACGATGACGA-3' and 5'-Pi-ATCGTTCGTC-3', in the presence of stopper-forming oligonucleotides, 5'Pi-ATCGTAAGATCGGCAGTTCCTTGCG-3' and 5'CGCAAGGAACTGCCGATCTT-3`. The synthesis was conducted as described in Figure S5. The synthesized molecules were separated using ion-exchange HPLC (as shown in Figure S6). The average length of the molecules was $\sim 850 \mathrm{~nm}$ (which corresponds to $\sim 2.5 \mathrm{kbp}$ ). B - AFM image of linearized Puc19 plasmid (2686 bases). C - Size-exclusion HPLC chromatography of the synthesized DNA (brown curve), the plasmid (green curve), $150 \mathrm{bp}$ poly $(\mathrm{dG})$-poly(dC) (blue curve) and 500 bp poly(dG)-poly(dC) (red curve) under alkaline conditions. The HPLC separation and AFM imaging were conducted as described in Materials and Methods. 

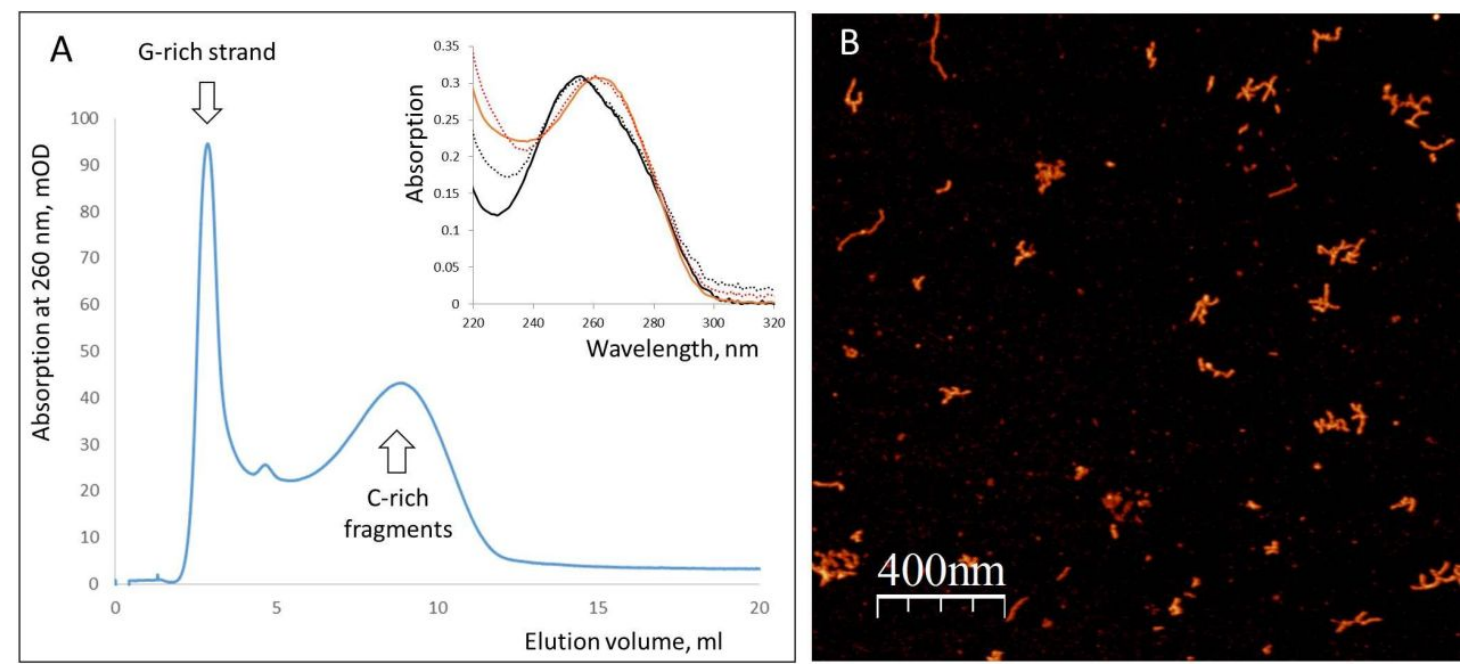

Figure S9. HPLC separation and AFM analysis of long single-stranded telomeric DNA. DNA molecules were synthesized from a pair of half-complementary hexameric oligonucleotides, $5{ }^{\circ}-\mathrm{Pi}-\mathrm{GTTAGG}-3^{\prime}$ and $5{ }^{\circ}-\mathrm{Pi}-\mathrm{AACCCT}-3^{\prime}$. The former oligonucleotide was more than $99.5 \%$ 5 '-end phosphorylated, while the latter contained $10 \%$ of the non-phosphorylated form. The DNA synthesis was conducted as described in Materials and Methods. A - Separation of the G-rich strand from the nicked $\mathrm{C}$-rich strand by size-exclusion chromatography on a Sepharose CL-2B column under alkaline conditions was as described in Materials and Methods. The inset shows the absorption spectra of: the void volume ("G-strand") fraction (solid black curve), 5-Pi-GTTAGG-3 (dotted black curve), "C-rich fragments" fraction (solid red curve), and 5'-Pi-AACCCT-3' (dotted red curve). B - AFM image of the G-rich strand prepared as described in "A". pH of the strand solution (eluted from the column in $0.1 \mathrm{M} \mathrm{LiOH}$ and $0.1 \mathrm{M} \mathrm{NaCl}$ ) was brought to neutral $\mathrm{pH}$ by dialysis against $5 \mathrm{mM} \mathrm{NaCl}$ for 16 hours. The molecules were deposited on mica and imaged as described in Materials and Methods. 


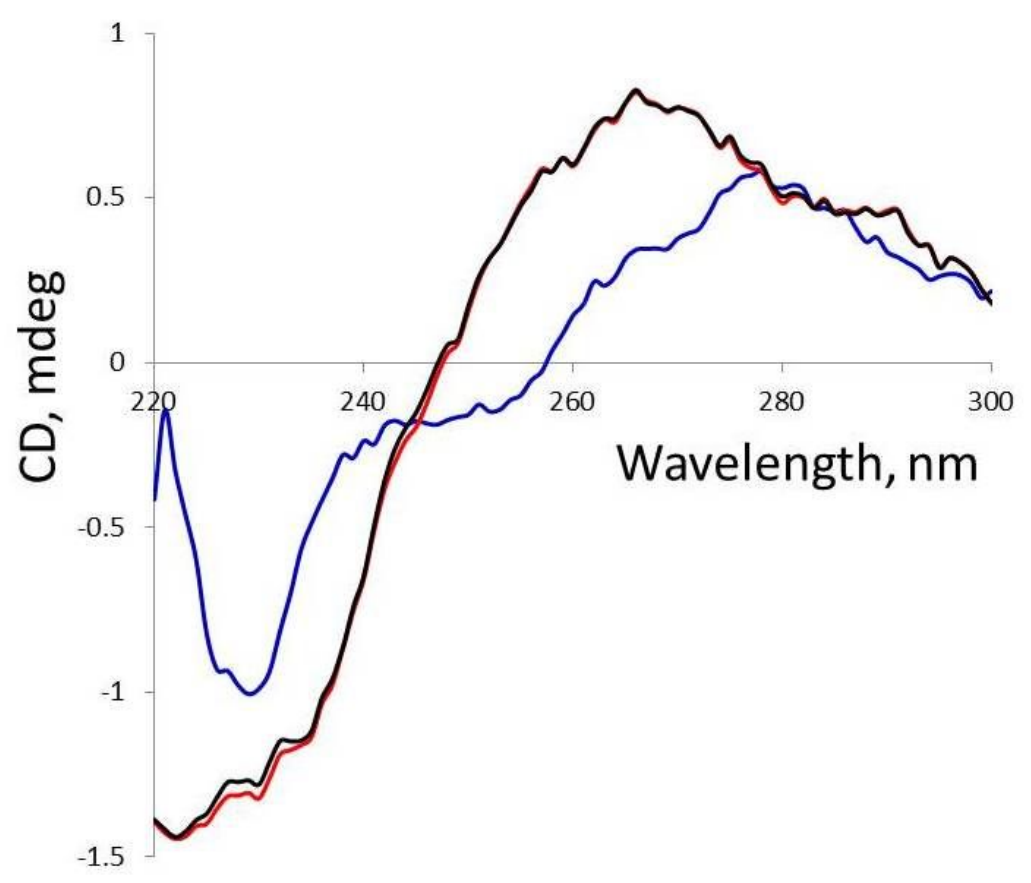

Figure S10. CD spectrometry of the telomeric strand. CD spectra of the G-rich strand prepared as described in Figure $\mathrm{S} 9$ in $2 \mathrm{mM}$ Tris-Acetate ( $\mathrm{pH} \mathrm{7.5)} \mathrm{(black} \mathrm{curve)} \mathrm{in} \mathrm{the}$ presence of $50 \mathrm{mM} \mathrm{KCl}$ (red curve), or in the presence of $0.1 \mathrm{M} \mathrm{LiOH}$ (blue curve). CD spectra were recorded at $25^{\circ} \mathrm{C}$ with a Chirascan V100 CD spectrometer (Applied Photophysics, UK) Each spectrum was recorded from 220 to $300 \mathrm{~nm}$ and was an average of 5 scans.
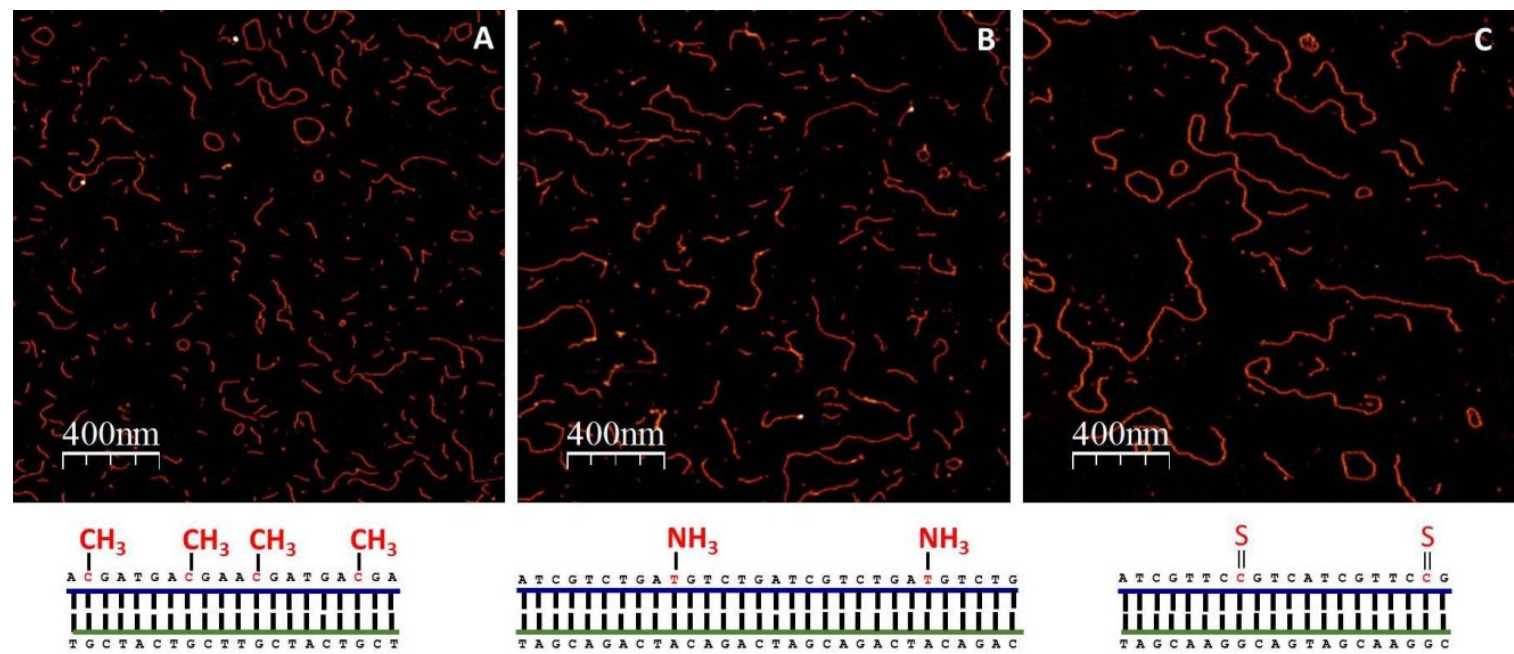

Figure S11. AFM images of long repeating dsDNA bearing modified residues. The molecules containing periodically repeated 5-methylcytosine (A), 5-amino-modified thymidine (B) or phosphorothioated nucleotide $(C)$ residues were synthesized from the following pairs of halfcomplementary oligonucleotides: '2' and '12'; ' 8 ' and '10' and '1' and '11' (see Table S1), respectively as shown in Materials and Methods. Schematic illustration of the synthesized molecules is given beneath each image. 


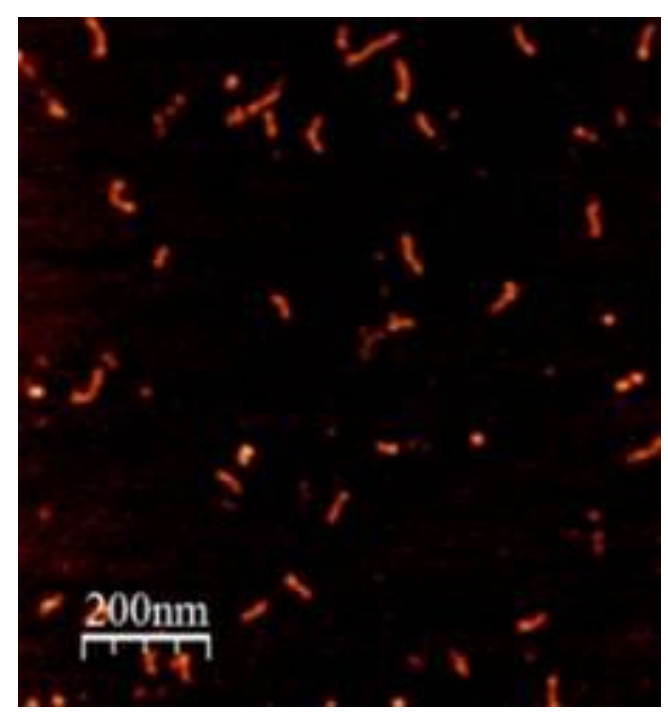

Figure S12. AFM image of long repeating dsDNA containing fluorescein-labeled nucleotides. The molecules were synthesized from a pair of half-complementary oligonucleotides: 5'-PiATCGTCTGA[T-Flu]GTCTG-3' and 5'-Pi-ACGATCAGACATCAG-3' as described in Materials and Methods. The former oligonucleotide containing an amino group was conjugated with FITC and subsequently purified by HPLC. The molecules were deposited on mica and imaged as described in Materials and Methods.
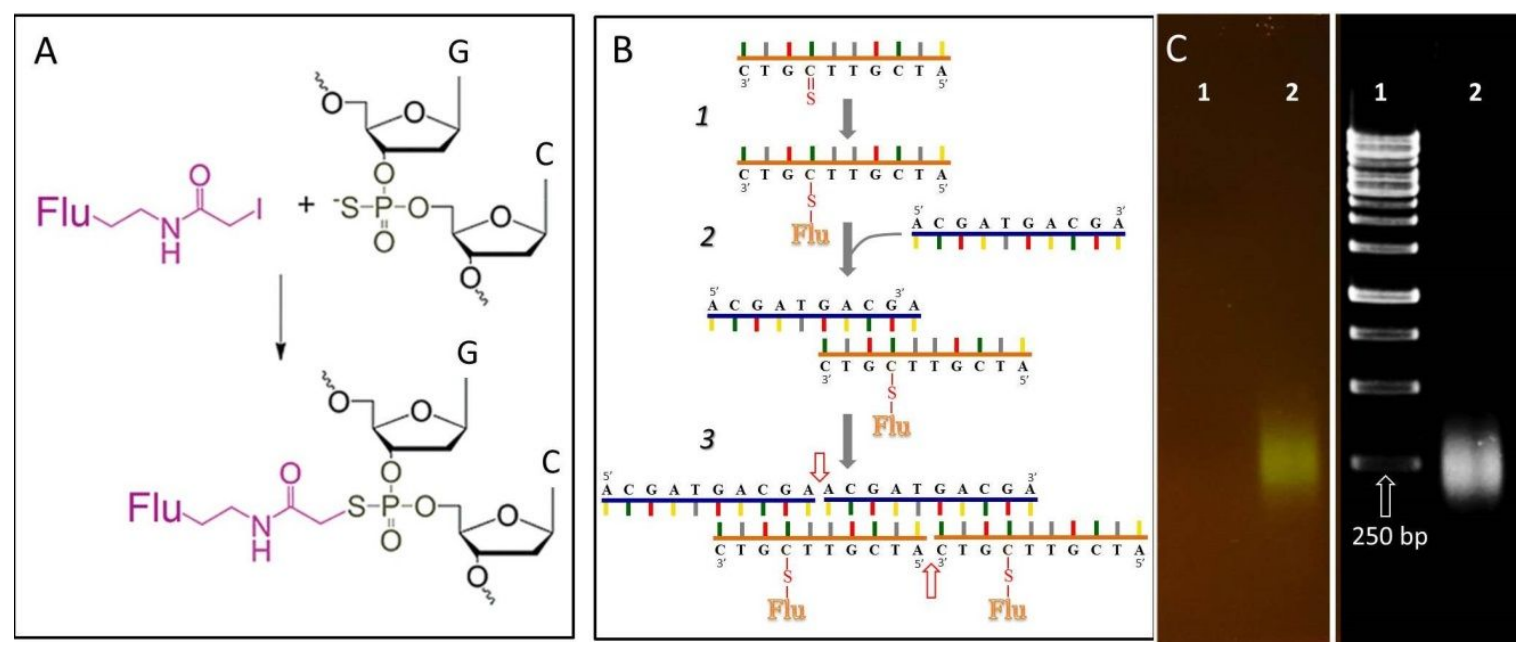

Figure S13. Synthesis of repeating dsDNA containing fluorescein residues attached to the nucleic acid backbone. The molecules were synthesized from a pair of half-complementary oligonucleotides: 5'-Pi-ATCGTT[C*-Flu]GTC-3' and 5'-Pi-ACGATGACGA-3' as described in Materials and Methods. The former oligonucleotide was prepared by incubation of 5 '-PiATCGTCTGA[C*']GTCTG-3' containing a phosphorothioated cytosine (marked with an asterisk) with iodoacetamide derivative of fluorescein (IAF). A - Schematic drawing of the reaction between IAF and the phosphorothioate group of the oligonucleotide. The conjugate 
between the dye and the oligonucleotide was purified by ion-exchange HPLC as described in Materials and Methods and incubated with an equimolar concentration of 5'-PiACGATGACGA-3' for 16 hours in the presence of DNA ligase as described in Materials and Methods. B - Schematic illustration half-complementary decameric oligonucleotides assembling into a fluorescein-labeled ds nucleic acid polymer. 1 - Attachment of a fluorescein residue (Flu) to the phosphorothioate group of the oligonucleotide; 2 Hybridization of the oligonucleotide with the half-complementary one; 3 - Binding of the dimers (formed in step 1) to each other. C - Agarose gel electrophoresis of the synthesized DNA. Electrophoresis was conducted at $1.5 \%$ agarose for 45 minutes at $130 \mathrm{~V}$. The gel was illuminated with Blue Light Transilluminator and the picture was taken by a digital camera (left panel); the gel was then stained with ethidium bromide and visualized with a Bio Imaging System 202D at $302 \mathrm{~nm}$ (right panel).
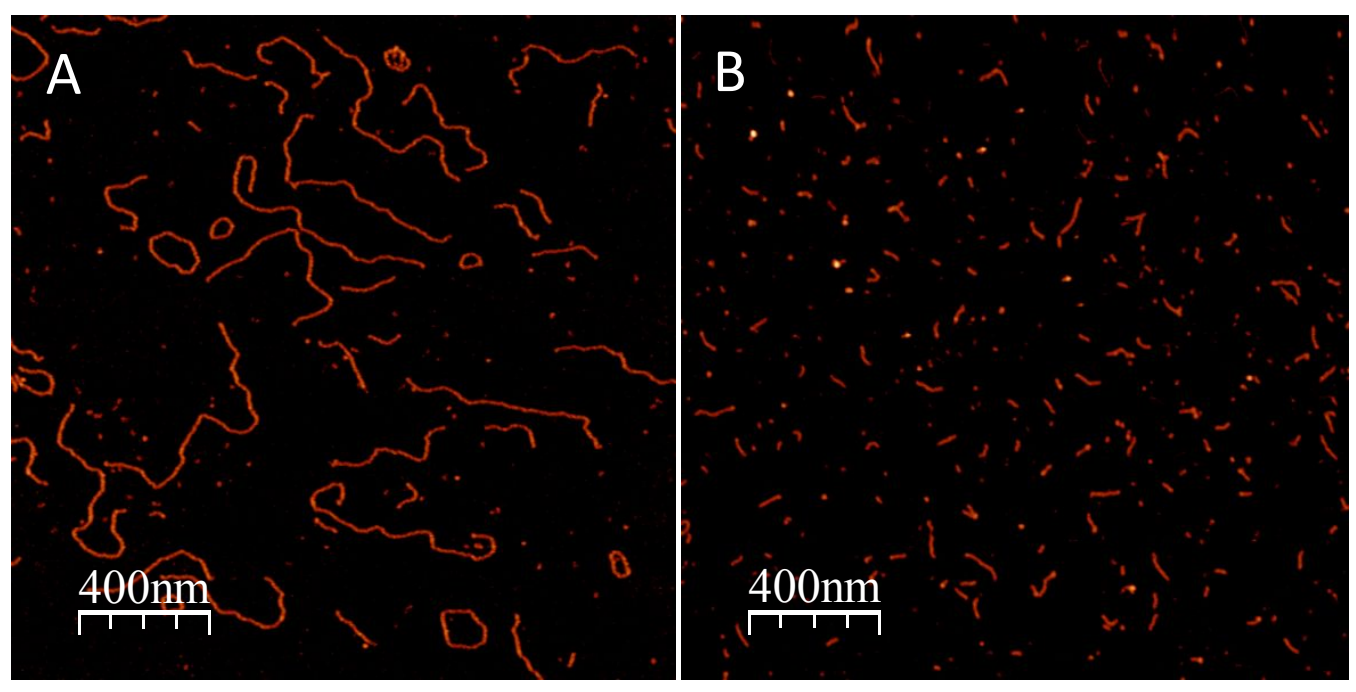

Figure S14. AFM images of repeating dsDNA containing fluorescein-labeled residues attached to the nucleic acid backbone. The molecules were synthesized as described in Fig. S13 from 5'-Pi-ATCGTT[C*]GTC-3' (A) or the fluorescein-labeled oligonucleotide, 5'-PiATCGTT[C*-Flu]GTC-3' (B) and 5'-Pi-ACGATGACGA-3' as described in Materials and Methods. The molecules were deposited on mica and imaged by AFM. 5'-Pi-ATCGTT[C*Flu]GTC-3' was prepared by modification of 5"-Pi-ATCGTT[C ${ }^{*}$ ]GTC-3' with IAF as described in Materials and Methods. The imaging analysis of more than 100 molecules showed that the average heights of the fluorescein-labeled $(B)$ and the non-labeled $(A)$ dsDNA are equal to $0.9 \pm 0.3$ and $0.8 \pm 0.2 \mathrm{~nm}$, respectively. . 